\title{
A Multi-Generation Sublethal Assay of Phenols Using the Nematode Caenorhabditis elegans
}

\author{
Nobuaki Tominaga, ${ }^{*}, a$ Shinya Kohra, ${ }^{b}$ Taisen Iguchi, ${ }^{c, d}$ and Koji Arizono ${ }^{c, e}$ \\ ${ }^{a}$ Department of Chemical and Biochemical Engineering, Ariake National College of Technology, 150 Higashi-hagio-machi, Omuta, \\ Fukuoka 836-8585, Japan, ${ }^{b}$ Faculty of Environmental Sciences, Nagasaki University, 1-14 Bunkyo-machi, Nagasaki 852-8521, Japan, \\ ${ }^{c}$ CREST, JST, Japan Science and Technology Corporation, 4-1-8 Honmachi, Kawaguchi, Saitama 332-0012, Japan, ${ }^{d}$ Department of \\ Bio-environmental Research, Center for Interactive Bioscience, National Institute for Basic Biology, 5-1 Higashiyama Myodaiji, \\ Okazaki, Aichi 444-8585, Japan, and ${ }^{e}$ Faculty of Environmental and Symbiotic Sciences, Kumamoto Prefectural University, 3-1-100 \\ Tsukide, Kumamoto 862-8502, Japan
}

(Received June 23, 2003; Accepted July 22, 2003; Published online July 23, 2003)

\begin{abstract}
The nematode Caenorhabditis elegans (C. elegans) was used for a multiple-generation toxicity bioassay of phenols. We examined the sublethal toxicity (fecundity and reproduction) of bisphenol A (BisA), 4-(1-adamantyl) phenol (Adp), and 4,4'-(1,3-adamantanediyl) diphenol (AdDP) over five generations using a Nematode Growth Medium (NGM) $1.7 \%$ agar plate. In the fourth generation, the phenols affected the fecundity rate of C. elegans at doses 100- to 10000 -fold lower than the $\mathrm{LC}_{50} \mathrm{~s}$. In particular, at $1 \mathrm{nM} \mathrm{BisA}$, the number of worms decreased significantly to about $50 \%$ of control $(p<0.05)$. A comparison of the number of viable worms and eggs suggested that the phenols exert hatching toxicity. In addition, individuals with an abnormality in the vulva, which could not ovulate, appeared on the phenol-containing plates. We confirmed that phenols disrupt reproduction in C. elegans and that the method using NGM agar plates facilitates multi-generational toxicity tests of chemicals that are poorly soluble in water.
\end{abstract}

Key words — Caenorhabditis elegans, reproduction, fecundity, reproductive disruption

\section{INTRODUCTION}

Recently, endocrine disrupting chemicals (EDCs) have received increasing attention from ecotoxicologists. ${ }^{1)}$ An unimaginable number of chemicals are released daily into the environment, and many of these chemicals are suspected to have endocrine-disrupting activity and thereby affect organisms in many ecosystems. Hence, there has been an increasing need for a rapid assay system to evaluate the toxicity of chemicals at lower concentrations.

Caenorhabditis elegans (C. elegans) is a freeliving nematode with a thin, transparent body approximately $1 \mathrm{~mm}$ in length, which lives mainly in the liquid phase of soils. The genome of this organism is completely mapped, ${ }^{2)}$ and it has become a commonly used laboratory model for molecular and cellular biology owing to its short life cycle (3 days

\footnotetext{
*To whom correspondence should be addressed: Department of Chemical and Biological Engineering, Ariake National College of Technology, 150 Higashihagio-machi, Omuta, Fukuoka 8368585, Japan. Tel.: +81-944-53-8747; Fax: +81-944-53-8758; Email: tominaga@ariake-nct.ac.jp
}

at $20^{\circ} \mathrm{C}$ ) and ease of rearing in the laboratory. ${ }^{3)}$ Many studies have used C. elegans in assessments of acute and chronic chemical hazards, ${ }^{4-14)}$ and thus $C$. elegans serves as an efficient model for testing the effects of xenobiotic chemicals in vivo. Accordingly, we have established several laboratory-scale bioassay systems to evaluate the impact of various chemicals on C. elegans. ${ }^{15-18)}$

Nonylphenol (NP) and bisphenol A (BisA) are known to bind estrogen receptors. Yamakoshi et al. reported previously that 4-(1-adamantyl) phenol (AdP) and 4,4'-(1,3-adamantanediyl) diphenol (AdDP) exhibited potent estrogenic activity. ${ }^{19)}$ In the present paper, we investigated the sublethal effects of BisA, AdP, and AdDP over five generations using fecundity, reproduction, and physical abnormality as toxicity endpoints.

\section{MATERIALS AND METHODS}

Chemicals — Agar, dimethyl sulfoxide (DMSO), Tryptone, sodium chloride, and BisA were purchased from Wako Pure Chemicals (Japan). Reagent grade 
AdP and AdDP were supplied by Aldrich.

Animals and Culture Conditions - The wildtype N2 strain of $C$. elegans was used in the present study. Worms were maintained and handled as described by Brenner. ${ }^{20)}$ Briefly, 3 to 5 worms were grown on a nematode growth medium (NGM) $1.7 \%$ agar plate with a lawn of Escherichia coli (E. coli) DH5 $\alpha$ as a food source and incubated at $20^{\circ} \mathrm{C}$. Every 4 or 5 days, worms were sub-cultured to new plates.

Multi-Generation Test - DMSO was used as the toxicant vehicle at a final concentration of $0.5 \%{ }^{17)}$ The test chemicals in DMSO were added to autoclaved NGM 1.7\% agar medium. After the mixture was sonicated, the warm medium was poured into the petri plates and allowed to harden. Subsequently, E. coli DH5 $\alpha$ was spread on the plate. Approximately 20 C. elegans eggs, obtained by alkalibleaching method, ${ }^{5)}$ were placed on each plate and incubated at $16^{\circ} \mathrm{C}$. One L4 larva was then transferred to a separate plate and defined as the first generation. Four replicates were set up for each treatment. The worms and eggs on each plate were counted under a dissecting microscope at a fixed time every day. The day that the first offspring was identified was defined as the first day. When the second generation worms attained the L4 stage, one worm was sub-cultured to a new plate. The above steps were repeated until the fifth generation was cultured. These experiments were carried out at least twice.

Statistical Analyses — Where applicable, data are reported as the mean \pm S.E.M. Statistical significance was evaluated by analysis of variance (ANOVA). Values of $p<0.05$ were considered statistically significant.

\section{RESULTS AND DISCUSSION}

Previously, we demonstrated that an acute toxicity assay using C. elegans as a model organism is a convenient and easy method for evaluation of chemical substances. ${ }^{17,18)}$ Others have also proposed the use of $C$. elegans for evaluating aquatic or soil toxicity. However, water insoluble or slightly soluble chemicals could not be evaluated in many cases because of the experimental conditions.

Previously, we reported that $C$. elegans responded to specific steroids in reproduction and fecundity rates. ${ }^{21)}$ In that experiment, C. elegans were exposed to various steroids on an agar plate. In the present paper, we investigated the applicability of
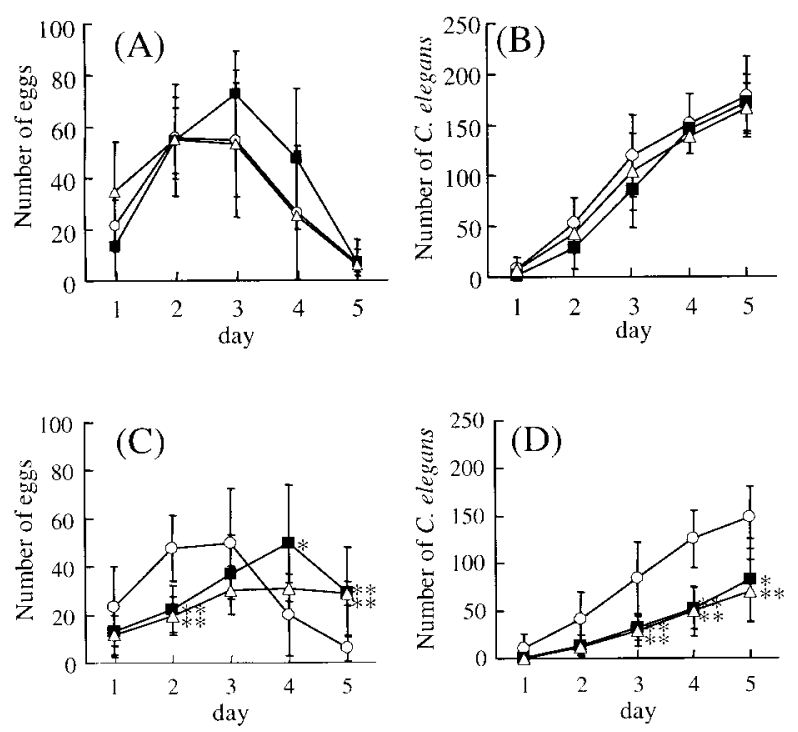

Fig. 1. Effects of Bis A on the Fecundity of C.elegans

(A) Egg abundance at 1st generation, (B) C. elegans abundance at 1 st generation, (C) egg abundance at 4 th generation, (D) C. elegans abundance at 4 th generation. $\bigcirc$, Control; $\mathbf{\square}, 1 \mathrm{nM} ; \triangle, 10 \mathrm{nM}$.

our test system to low solubility or suspected endocrine disrupting chemicals. We tested three phenols, BisA, AdP, and AdDP. Figure 1 summarizes the results of the assay using C. elegans exposed to BisA on a NGM $1.7 \%$ agar plate containing $0.5 \mu \mathrm{M}$ cholesterol. First generation and control worms produced a similar number of eggs [Fig. 1(A)]. In contrast, exposure to BisA delayed the peak egg abundance [Fig. 1(C)]. The fertilized egg of C. elegans is an ellipsoid shape because of cell polarity, whereas an unfertilized egg is globular in shape. The shape of ovulated eggs in these exposures suggested that few were fertilized.

Worm abundance during the first generation did not differ significantly from controls [Fig. 1(B)]. However, by days 3-5 of the fourth generation, abundance had decreased to about $50 \%$ of controls [Fig. 1(D)]. The abundance of viable worms and eggs in these exposures suggested that BisA exerts toxic effects on hatching. Similar effects were observed in response to nonylphenol. ${ }^{16)}$ The BisA 50\% lethal concentration $\left(\mathrm{LC}_{50}\right)$ value for C. elegans was over $100 \mu \mathrm{M} .^{18)}$ In our experiments, sublethal effects of BisA on C. elegans were observed at 1 and $10 \mathrm{nM}$, levels that are over 10000-fold lower than the $\mathrm{LC}_{50}$ value. On $100 \mathrm{nM}$ BisA plates, C. elegans lost fecundity, and we could not culture them after the third generation.

The effects of AdP on C. elegans fecundity are shown in Fig. 2. AdP exposure affected egg abun- 

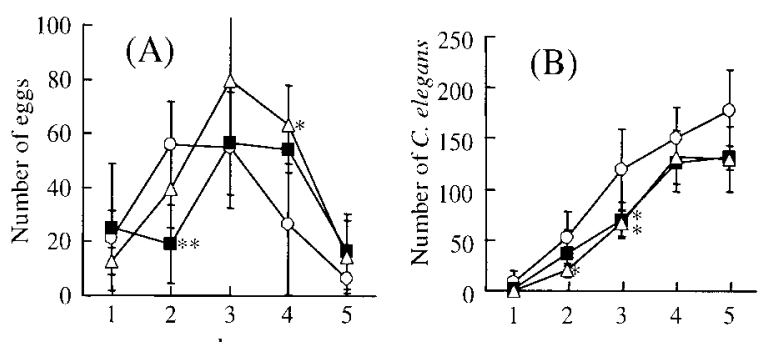

day


Fig. 2. Effects of AdP on the Fecundity of C. elegans

(A) Egg abundance at 1st generation, (B) C. elegans abundance at 1st generation, (C) egg abundance at 4th generation, (D) C. elegans abundance at 4 th generation. $\bigcirc$, Control; $\mathbf{\square}, 10 \mathrm{nM} ; \triangle, 100 \mathrm{nM}$.

dance patterns, and the effects on the fourth generation were more severe than on the first generation [Figs. 2(A) and 2(C)]. The abundance of worms in the first generation decreased by about $15 \%$ from the control levels at $10 \mathrm{nM}$ or $100 \mathrm{nM}$ AdP [Fig. 2(B)], and AdP exerted its effects faster than BisA. Moreover, at $10 \mathrm{nM} \mathrm{AdP,} \mathrm{the} \mathrm{fourth} \mathrm{genera-}$ tion of viable worms decreased to about $40 \%$ of control $(p<0.01)$ [Fig. 2(D)]. Our research showed that exposure to greater than $10 \mathrm{nM}$ of AdDP decreased fecundity to the same extent as AdP (Figs. 2 and 3). However, the effects of high concentration and low concentration exposures to AdDP did not differ significantly and a dose-response relationship was not observed. Chu et al. ${ }^{14)}$ reported that dose-response relationship was not observed in some case of lethal toxicity of metals on C. elegans. The dose response curves of copper and zinc in mortality rate on $C$. elegans did not show the dose dependent manner at low concentration. We were unable to demonstrate reproductive toxicity below $1 \mathrm{nM}$ BisA, AdP, or AdDP using this NGM 1.7\% agar plate bioassay system.

We previously reported that worms occasionally exhibited an abnormal vulva when exposed nonylphenol. ${ }^{16)}$ In the present study, in all exposed groups also occasionally individuals exhibited an abnormality in the vulva (Fig. 4). Greenwald et al. reported that lin-12(null) mutant, lin-12(n137 n720), had a large protrusion at the normal position of the
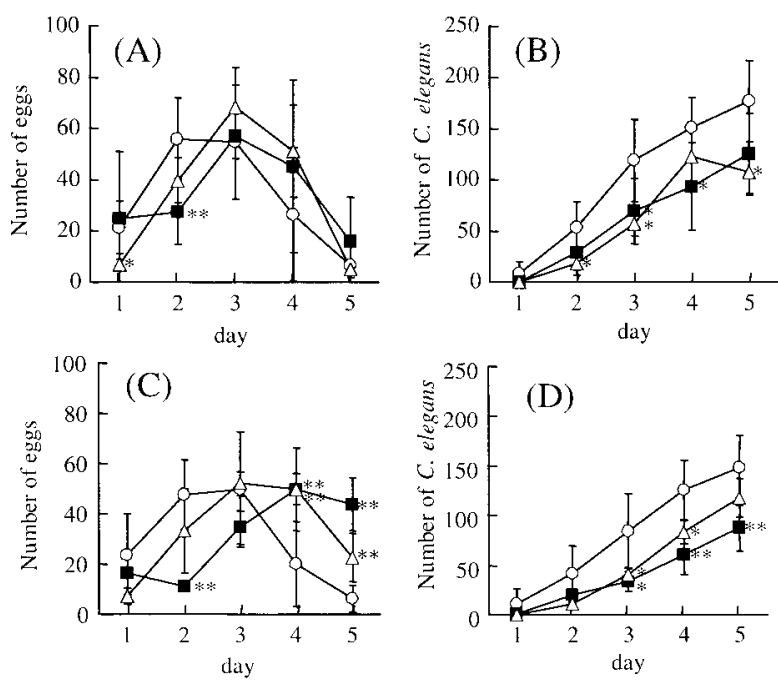

Fig. 3. Effects of AdDP on the Fecundity of C. elegans

(A) Egg abundance at 1st generation, (B) C. elegans abundance at 1st generation, (C) egg abundance at 4th generation, (D) C. elegans abundance at 4 th generation. $\bigcirc$, Control; $\mathbf{\square}, 10 \mathrm{nM}, \triangle, 100 \mathrm{nM}$.

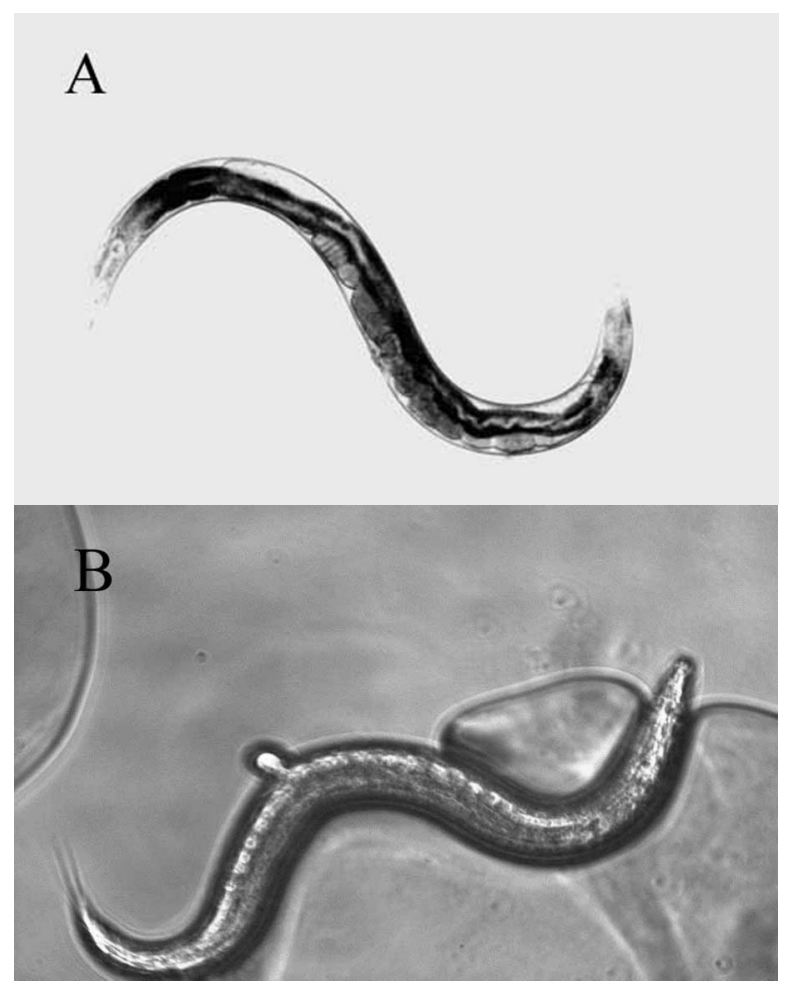

Fig. 4. Photographs of Normal Hermaphrodite (A) and Individual with Large Protrusion at the Normal Position of the Vulva (B), which was Exposed to AdP

vulva $^{22)}$ similar to the result of our experiment. The lin-12 is a gene that specifies the fates of cells with fates controlled by cell interaction. These facts and our results suggest that phenolic chemicals may ex- 
ert disruptive effects on fates of cell and effect on reproduction. We have been used cDNA microarrays to explore the variation in expression of approximately 5000 unique genes of $C$. elegans exposed chemicals including BisA. When the analysis is finished, more information is obtained and the point of application may be clarified.

Although BisA is nearly insoluble in water (BisA 120-300 mg/l), the NGM $1.7 \%$ agar plates appear to provide an effective vehicle for multi-generational toxicity tests using water-insoluble chemicals.

In conclusion, we examined the effects of BisA, $\mathrm{AdP}$ and AdDP on C. elegans over five generations and clarified their sublethal toxicity at a concentration of about $1 / 100$ to $1 / 10000$ of $\mathrm{LC}_{50}$ using fecundity, reproduction, growth and physical abnormality as toxicity endpoints. And the chemicals appear to have some hatching toxicity.

Acknowledgements The authors thank Mr. H. Yokoo and Ms. K. Komiya, Ariake National College of Technology, for their skillful technical assistance. Support for this study was provided in part by grants from the Japan Society for the Promotion of Science (Grant-in-Aid for Scientific Research (C) 13833009) and from the Japan Ministry of Education, Culture, Sports, Science and Technology (Grant-in-Aid for Scientific Research on Priority Areas (1) 14042265) to N. T. and CREST to K. A.

\section{REFERENCES}

1) Colborn, T., Dumanoski, D. and Myers, J. P. (1996) Our stolen future, Dutton, New York.

2) The C. elegans sequencing consortium (1998) Genome sequence of the nematode $C$. elegans: A platform for investigating biology. Science, 282, 20122018.

3) Sulston, J. and Hodgkin, J. (1988) Methods. In The nematode Caenorhabditis elegans (Wood, W. B., ed.), Cold Spring Harbor Laboratory, Cold Spring Harbor, NY, U.S.A., pp. 587-606.

4) Ohba, K. and Ishibashi, N. (1984) A nematode, Caenorhabditis elegans, as test organism for nematicide evaluation. J. Pesticide Science, 9, 91-96.

5) Williams, P. L. and Dusenbery, D. B. (1990) Aquatic toxicology testing using the nematode, Caenorhabditis elegans. Environ. Toxicol. Chem., 9, 1285-1290.

6) Donkin, S. G. and Dusenbery, D. B. (1993) Soil toxicity test using the nematode Caenorhabditis elegans and an effective method of recovery. Arch. Environ. Contam. Toxicol., 25, 145-151.

7) Traunspurger, W., Haitzer, M., Hoss, S., Beier, S., Ahlf, W. and Steinberg, C. (1997) Ecotoxicological assessment of aquatic sediments with Caenorhabditis elegans (nematoda)-A method for testing liquid medium and whole-sediment samples. Environ. Toxicol. Chem., 16, 245-250.

8) Khanna, N., Cressman III, C. P., Tatara, C. P. and Williams, P. L. (1997) Tolerance of the nematode Caenorhabditis elegans to $\mathrm{pH}$, salinity, and hardness in aquatic media. Arch. Environ. Contam. Toxicol., 32, 110-114.

9) Dhawan, R., Dusenbery, D. B. and Williams, P. L. (1999) Comparison of lethality, reproduction, and behavior as toxicological endpoints in the nematode Caenorhabditis elegans. J. Toxicol. Environ. Health A, 58, 451-462.

10) Power, R. S. and de Pomerai, D. I. (1999) Effect of single and paired metal input in soil on a stress inducible transgenic nematode. Arch. Environ. Contam. Toxicol., 37, 503-511.

11) Peredney, C. L. and Williams, P. L. (2000) Utility of Caenorhabditis elegans for assessing heavy metal contamination in artificial soil. Arch. Environ. Contam. Toxicol., 39, 113-118.

12) Mori, T., Mohamed, A. S. A., Sato, M. and Tamasaki, T. (2000) Ellagitannin toxicity in the free-living soilinhabiting nematode, Caenorhabditis elegans. J. Pesticide Science, 25, 405-409.

13) Anderson, G. L., Boyd, W. A. and Williams, P. L. (2001) Assessment of a sublethal endpoint for toxicity testing with the nematode Caenorhabditis elegans. Environ. Toxicol. Chem., 20, 833-838.

14) Chu, K. W. and Chow, K. L. (2002) Synergistic toxicity of multiple heavy metals is revealed by a biological assay using a nematode and its transgenic derivative. Aquat. Toxicol., 61, 53-64.

15) Kohra, S., Kuwahara, K., Takao, Y., Ishibashi, Y., Lee, H. C., Arizono, K. and Tominaga, N. (2001) Effect of Bisphenol A on the feeding behavior of Caenorhabditis elegans. J. Health Sci., 48, 93-95.

16) Tominaga, N., Tomoeda, M., Kohra, S., Takao, Y., Nagae, M., Ueda, K., Ishibashi, Y., Kai, T. and Arizono, K. (2002) A convenient sublethal assay of alkylphenol and organotin compounds using the nematode Caenorhabditis elegans. J. Health Sci., 48, 555-559.

17) Tominaga, N., Kunimoto, M., Kai, T., Arizono, K. and Kohra, S. (2003) A convenient assay for evaluating chemical toxicity using Caenorhabditis elegans as a model organism. Environ. Sci., 10, 215221.

18) Ura, K., Kai, T., Sakata, S., Iguchi, T. and Arizono, 
K. (2002) Aquatic acute toxicity testing using the nematode Caenorhabditis elegans. J. Health Sci., 48, 583-586.

19) Nikov, G. N., Eshete, M., Rajnarayanan, R. V. and Alworth, W. (2001) Interactions of synthetic estrogens with human estrogen reseptors. J. Endocrinol., 170, 137-145.

20) Brenner, S. J. (1974) The genetics of Caenorhabditis elegans. Genetics, 77, 71-94.

21) Tominaga, N., Ura, K., Kawakami, M., Kawaguchi, T., Kohra, S., Mitsui, Y., Iguchi, T. and Arizono, K. (2003) Caenorhabditis elegans responses to specific steroid hormones. J. Health Sci., 49, 28-33.

22) Greenwald, I. S., Sternberg, P. W. and Horvitz, H. R. (1983) The lin-12 locus specifies cell fates in Caenorhabditis elegans. Cell, 34, 435-444. 